\title{
A STUDY OF LEARNING CURVE IMPACT ON THREE IDENTICAL SMALL SPACECRAFT
}

\author{
Guangming Chen, NASA Administrator's Fellow, Associate Professor \\ Dept. of Industrial, Manufacturing and Information Engineering \\ School of Engineering \\ Morgan State University \\ Baltimore, Maryland \\ Douglas D. McLennan, ST-5 Project Manager \\ Code 495 \\ Goddard Space Flight Center \\ Greenbelt, Maryland
}

\begin{abstract}
With an eye to the future strategic needs of NASA, the New Millennium Program is funding the Space Technology 5 (ST-5) project to address the future needs in the area of small satellites in constellation missions. The ST-5 project, being developed at Goddard Space Flight Center, involves the development and simultaneous launch of three small, 20-kilogram-class spacecraft. ST-5 is only a test drive and future NASA science missions may call for fleets of spacecraft containing tens of smart and capable satellites in an intelligent constellation. The objective of ST-5 project is to develop three such pioneering small spacecraft for flight validation of several critical new technologies. The ST-5 project team at Goddard Space Flight Center has completed the spacecraft design, is now building and testing the three flight units. The launch readiness date (LRD) is in December 2005. A critical part of ST-5 mission is to prove that it is possible to build these small but capable spacecraft with recurring cost low enough to make future NASA's multispacecraft constellation missions viable from a cost standpoint.

The resources spent on concept development and design can be characterized as "nonrecurring cost" because it is not needed for future units. Nonrecurring cost primarily depends on the mission requirement, and in the case of ST-5, the inherent technological complication. However, recurring cost, the effort and resource spent in building and testing every unit of spacecraft, may be significantly affected by the "learning curve effect", due to the complicated structure of spacecraft or subsystems, as well as the uniqueness of aerospace products. For multi-spacecraft missions, recurring cost is one of our main concerns. In this paper, we have conducted a research on the learning curve impact to the three ST-5 small satellites, including the impact to the entire spacecraft and the impact to primary subsystems, though a majority of ST-5 funding is contributed toward nonrecurring cost. This study is important and useful in assessment of NASA's future multi-spacecraft missions. It may help provide a more accurate estimate of the mission cost and give us an insight leading to possible cost reduction for the future multi-spacecraft missions.
\end{abstract}




\section{Introduction}

The resurgence of interest in small satellites is primarily due to the increasing demands for timely and affordable access to space. In fact, most of the satellites launched in early space age were small, due to the limited launch vehicle capacities at that time. With the much more complicated requirements of space missions today and the increasing capacities of launch vehicles, today's satellites are becoming larger and more expensive. The development span from concept to useful orbital operation usually takes several years. New ideas or technologies that need experimental validation in space environment, find it difficult to gain timely access to space $[1,2]$. To validate the new ideas and technologies in space at affordable costs, it is strategically necessary to explore the novel design and production methodologies for smaller and less expensive satellites.

To achieve the goal of making inexpensive spacecraft, combined with the growing financial pressures, NASA has become interested in the usage of small satellites to complement large ones for many space applications. Indeed, NASA's strategic New Millennium Program for technological development of future space missions includes Space Technology 5 (ST-5) Small Satellite Constellation Theme. The ST-5 project involves the development of three small 20kilogram-class spacecraft, and launches them by one launch vehicle to test many new technologies. The continuous effort for future missions may call for fleets of spacecraft containing tens or more similar small satellites as a constellation, and operate them in multispacecraft science missions. This constellation could make multiple remote and in-situ measurements simultaneously in space, thus revolutionizing the scientific investigations of key physical processes explored by the NASA Space Science and Earth Science Enterprises. Goddard Space Flight Center (GSFC), located in Greenbelt, Maryland, has been assigned the responsibility to implement this pioneering mission [3]. ST-5 is a pathfinder for highly capable, low-cost small spacecraft, miniaturized subsystems, and constellation mission operations. ST-5 fabrication, assembly, test and operations strategies for multiple small satellites are critical to the success of future missions. Specifically, ST-5 project will validate many new technologies and the mission features include:

- Flight validation of breakthrough technologies;

- Validation of small spacecraft for research-quality scientific measurements of Earth's magnetic field;

- Constellation mission operations;

- Three $25 \mathrm{~kg}$ satellites, approximately $60 \mathrm{~cm} \times 30 \mathrm{~cm}$;

- Low power bus; High density avionics;

- High efficiency solar array and lithium-ion battery

- Digital low-power transponder

In addition, another critical part of the ST-5 mission is to prove that it is possible to build these small but capable spacecraft with a recurring cost low enough to make the future constellation missions viable from a cost standpoint. Satellite "production" usually consists of a unique, individual and "one of a kind" product. Mass production of satellites refers to a level of tens or hundreds. ST-5 project considers the development of three modules as a pathfinder. Novel design and manufacturing strategies for such a development is based upon the low-cost 
benefit of "mass production" and nonlinear effects of "learning curves". The study of learning curve impact on the recurring cost for ST-5 project is important, which can help develop a "road map" to the low-cost small satellites under multiple production runs of identical units, such as the manufacturing cost of lower than $\$ 1$ million per satellite. The development of the learning curve effects on the first three ST-5 units can help estimate the manufacturing cost for the identical or similar satellites of future production runs.

\section{ST-5 Trailblazer Spacecraft and Project Milestones}

The ST-5 project deals with fabrication of three pioneering $25-\mathrm{kg}$ small satellites. The task involves the development and investigation of ten to twenty kilogram-class spacecraft and their operation in multi-spacecraft science missions. It will support the vision of flying tens to hundreds of nano satellites in a constellation to make multiple remote and in-situ measurements simultaneously in space, thus revolutionizing the scientific investigations of key physical processes explored by the NASA Space Science and Earth Science enterprises. Ideas and models will be recorded for future missions that may eventually call for 100 identical spacecraft at a very low recurring cost. The conceptual development of the proposed pathfinders gives the basic overview on the deployed overall dimensions as well as the basic architectural block diagram. The spacecraft platform is spin stabilized with body mounted solar arrays and the spacecraft hardware concept layout is as shown in Figure 1. The basic structure is an octagonal prism, selected for its application to volume efficient multiple units packaging in launch vehicle for future missions. It is $20.0 \mathrm{~cm}$ in height by $41.6 \mathrm{~cm}$ in diameter across the flat facets $(44.0 \mathrm{~cm}$ including the stowed magnetometer) and $45.0 \mathrm{~cm}$ across the vertices. The overall height in the launch configuration is $29.0 \mathrm{~cm}$ from the base of the balance weights to the top of the X-band antenna. On orbit, the magnetometer instrument is deployed $59.0 \mathrm{~cm}$ from the spacecraft body.

Currently, the ST-5 project team has completed preliminary design and detailed design for all three identical flight units. The team is working hard to build, integrate and test the subsystems and entire flight units, aiming at a Launch Readiness Date (LRD) in December 2005. Some of the project milestones are given as follows:

- NASA Headquarters selects Nano-Satellite Constellation Trailblazer as ST-5 - August 1999;

- Successful System Concept Review (SCR) - May 10-11, 2000;

- Successful Preliminary Design Review (PDR) - June 19-20, 2001;

- Successful Confirmation Review - November 30, 2001;

- Successful Critical Design Review (CDR) - June 25-26, 2002;

- LRD - December 2005.

The project team consists of several subgroups: project and mission management under work breakdown structure (WBS) code 1.0; systems engineering under WBS code 2.0; spacecraft and subsystems under WBS code 3.0; ground system under WBS code 4.0 and 6.0; launch vehicle under WBS code 5.0. 


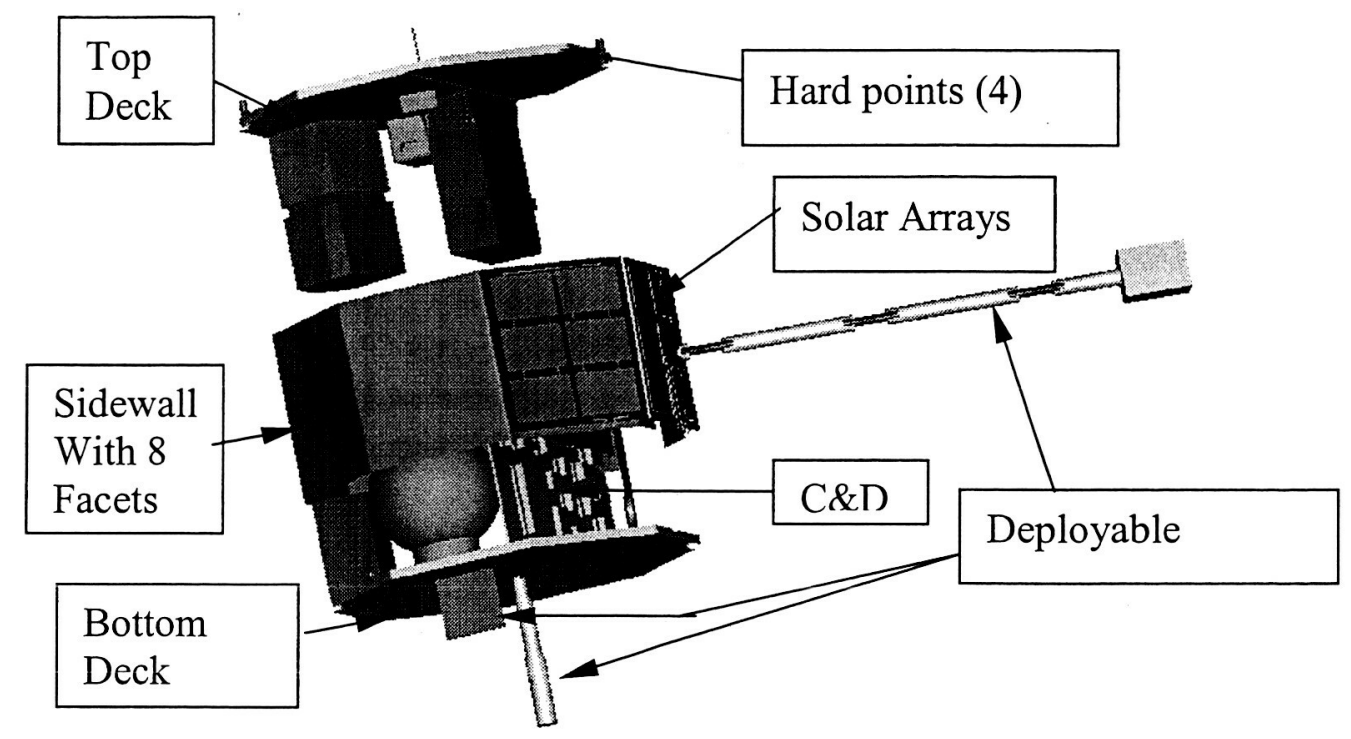

Figure 1. ST-5 Spacecraft Concept Layout

\section{Overall Nonrecurring Cost and Recurring Cost}

A large portion of the ST-5 cost is nonrecurring. Nonrecurring costs are one-time expenditures, generally including the costs and labors spent in the earlier stages in the space mission life cycle, such as the concept exploration effort, preliminary design and detailed design. Reference [4] divides the space mission life cycle as a basic 4-phase domain: concept exploration, detailed development, production and deployment, operations and support. Different missions may name the four stages by various terminologies but the tasks should be similar. Basically, majority of the costs spent in the first two stages can be categorized as nonrecurring. Also, a part of cost spent in later stages may also be categorized as nonrecurring, such as the expenditures on tooling, special manufacturing equipment and test instruments, as well as the setup cost of a production process. Regardless of this, however, most of the expenditures and labors spent on later stages of a space mission cycle should be considered as recurring, especially those on manufacturing, integration and testing, and mission operation, etc. Due to the manufacturing nature of small quantities of parts or subsystems in space missions and the requirement of highly-skill and technology-intensive labors, the learning curve has a significant nonlinear effect on the recurring cost.

The ST-5 team members have spent tremendous efforts in concept development, preliminary design, detailed design and the technology valuations. The costs spent in the first three years are basically considered as nonrecurring costs. The resources mainly consist of the expenditures on the first spacecraft in concept development, preliminary design and detailed design. These costs should be considered nonrecurring in nature, because they are one-time expenditure and not needed for the future units if more identical spacecraft are fabricated. The data of dollar amount in this paper is analyzed as a percent of the total cost, unless otherwise specified. 


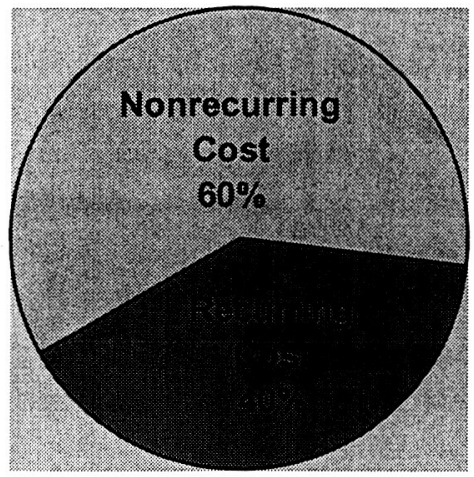

$\square$ Nonrecurring Cost

Recurring Cost

Figure 2. Preliminarily estimated nonrecurring cost and recurring cost of ST-5

Our analysis, together with the proposed estimates given by subsystem leads, indicates that around $60 \%$ of the expenditures can be categorized as nonrecurring and around $40 \%$ can be categorized as recurring (see the pie chart in Figure 2). Upon the completion of the project, the actual data on expenditures will be categorized more accurately. Also, the expenditure on the first flight unit is about $82 \%$ of the total expenditures, including a large portion of the nonrecurring cost. The expenditures for Flight Unit 2 and Flight Unit 3 are slightly different as given in Figure 3. To understand intuitively the learning curve effect on the recurring cost, we have reorganized the data as nonrecurring and recurring for unit 1, recurring for unit 2 and unit 3 , as given in the bar chart in Figure 4. Since the expenditures are preliminary estimates, the actual spending including the launch vehicle slip will be carefully categorized after the ST-5 mission is completed or nearly completed. The more accurate data will be utilized to estimate our model parameters. The established model could be a useful tool to predict the recurring cost for the possible tens of spacecraft constellation missions in the future, if multiple production runs of identical satellites are implemented.

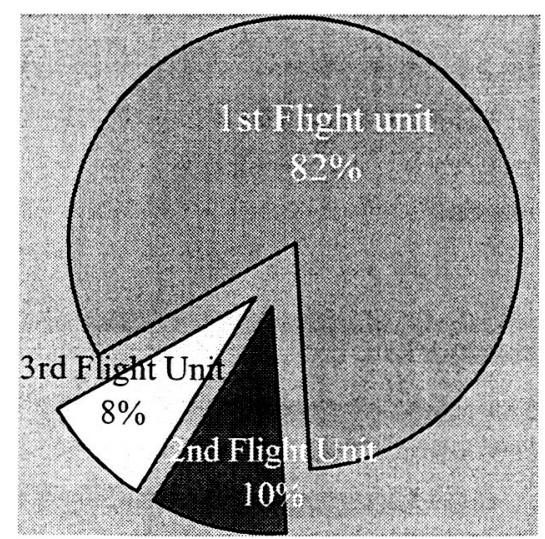

$\square$ 1st Flight unit
$\square$ 2nd Flight Unit
$\square$ 3rd Flight Unit

Figure 3. Comparison of the preliminarily predicted costs on three ST-5 Spacecraft (unit 1 includes nonrecurring cost). 


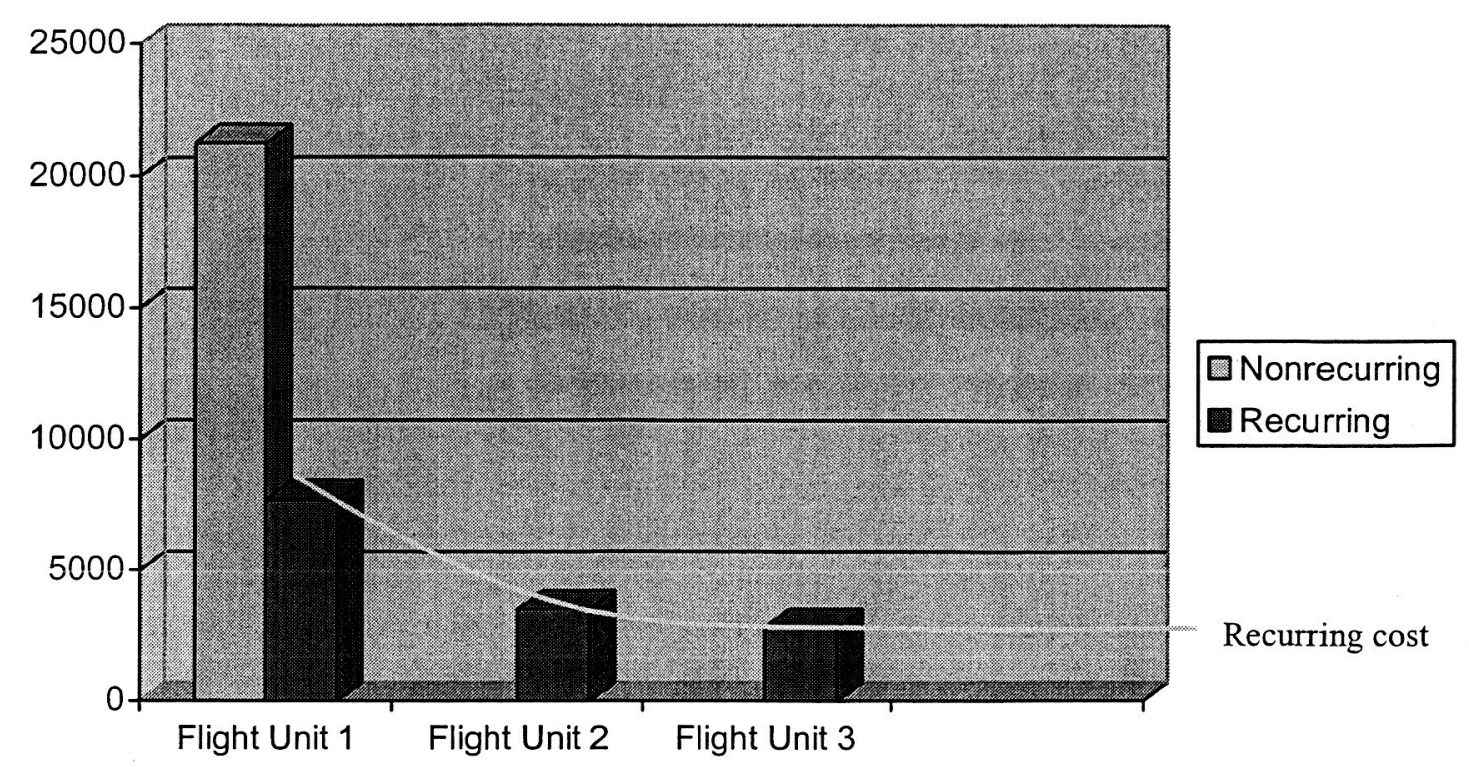

Figure 4. Nonrecurring and recurring cost for the three spacecraft. (coded data in $\$ 1000$ )

\section{Strategic Cost Reduction}

Recently, the space missions have been challenged for cost reduction while keeping highquality manufacturing standards. More versatile, sophisticated and lighter satellites must be produced at lower cost. Satellite production usually consists of a unique, individual and one of a kind product. On the contrary, mass production of satellites refers to some tens or hundreds. The ST-5 project considers the development of three modules as a pathfinder. Novel design and manufacturing strategy for such a development is based upon the cost-effective benefit of mass production and nonlinear effects of learning curve. Incorporating new design concepts with the traditional technologies is also a focus in the effort towards accomplishing this goal. The objective is not merely to design and launch a successful satellite, but also to reduce its cost. Consequently, we should probe the area for new methodology and new technological application, rather than just look at cost reductions in a conventional way. Theoretically, the total cost (TC) for mass production, can be estimated by a linear function:

$$
\mathrm{TC}=\mathrm{a}+\mathrm{bn}
$$

Where $\mathrm{a}$ is the initial setup or fixed cost to start a production run; $\mathrm{b}$ is the variable cost (labor, material, energy, etc.), recurring for each unit of product; $n$ is the number of products made [5]. This model may be used for the cost estimation for multiple production runs of identical ST-5 satellites. Setup cost is one-time expenditure to initialize the production and not recurring for each produced unit. This cost can refer to those for design configuration changes, manufacturing preparation and molds, equipment, tool and fixtures for multiple production runs. If NASA decides to expand the mission to a constellation involving 100 identical small satellites, Equation (1) may be used to estimate the production cost. As discussed before, nonrecurring cost is onetime expenditure and unnecessary for the $2^{\text {nd }}, 3^{\text {rd }}$ or repetitive units. Recurring cost refers to those repetitive costs for each piece of subsystem or product, including standard costs like labor 
and material cost. Due to the inherent technological complication of satellites and the nature of intensive high-tech skills, recurring cost $\mathrm{b}$ may be significantly affected by a nonlinear learning curve for the first several units produced. After the labor skills reach a sufficient level, the recurring cost for later units will primarily remain as a constant amount called standard variable cost $\mathrm{C}_{0}$. The reduction in recurring cost can be graphically seen in Figure 5.

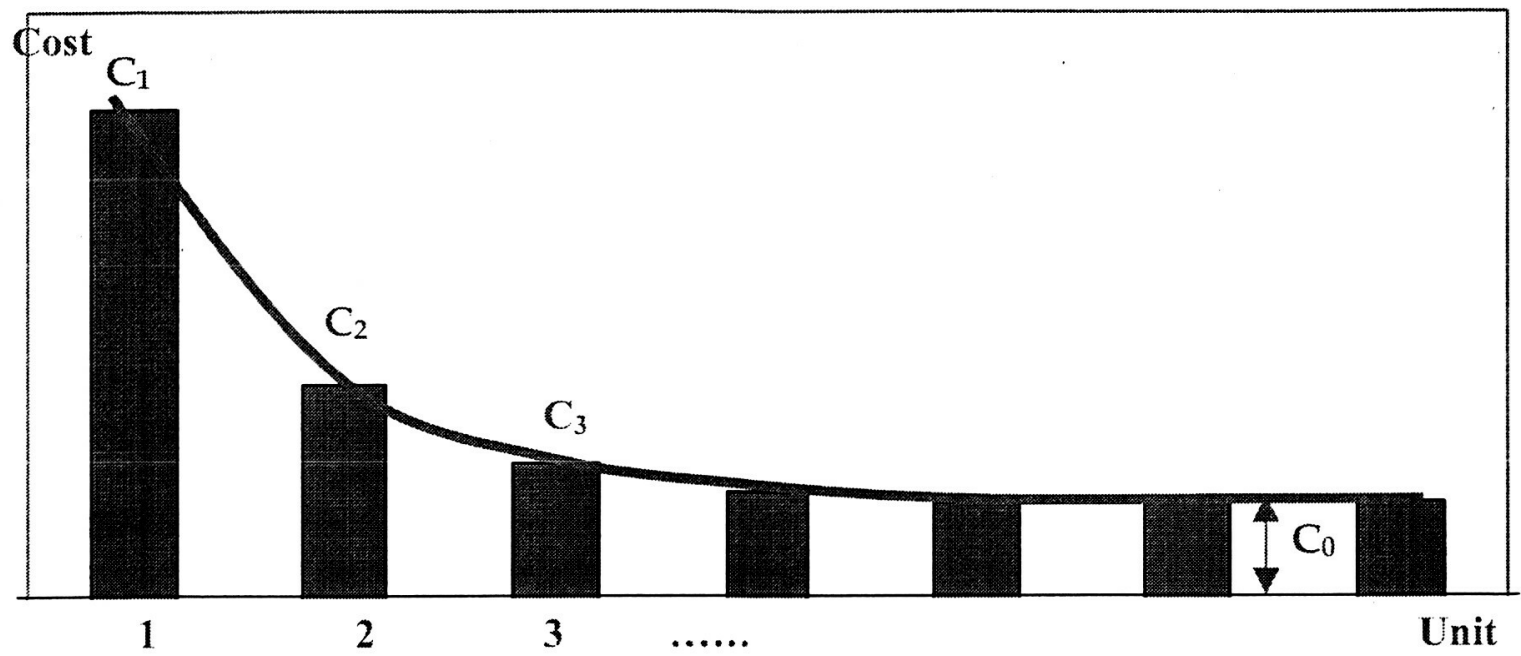

Figure 5. Learning Curve Effect on Recurring Manufacturing Cost of ST-5 Spacecraft

This learning curve effects model can be mathematically derived as bellow. The learning curve was adapted from the historical observation on the fact that individuals performing repetitive tasks exhibit an improvement in performance as the task is repeated a number of times [6]. Empirical studies of this phenomenon yields three conclusions on which the current theory and practice may be based upon:

1) The resources needed to learn how to do a task or make a unit (either entire spacecraft or subsystems) decreases as the same unit is repetitively fabricated;

2) The decreasing rate of needed "learning" resources reduces (or less improvement in cost) proportionally as more units are produced; For instance, net reduction in "learning" resources for $2^{\text {nd }}$ unit from the first unit should be larger than the net reduction for $32^{\text {nd }}$ unit from $31^{\text {st }}$ unit;

3) The standard resources needed to make the repetitive units will be a constant value $\mathrm{C}_{0}$ as specified in Figure 5 after the skills reach a sufficient level.

The consistency in improvement (or cost reduction) has been found existing in the form of a constant percentage reduction in the time required over successively quantities of units produced, which can be observed graphically in Figure 5. In fact, the mathematical model can be developed as: Define $C_{t}$ as the resource or cost required for unit $t$ product or subsystem produced $(t=1,2,3$ $\ldots)$; So, $C_{1}$ the cost required for the first unit. The derivative of $C_{t}$ with respect to $t\left(d_{t} / d t\right)$ is the reduction rate in cost required for unit $t$. In industrial application of developing learning curves, 
we can assume $\mathrm{dC}_{t} / \mathrm{dt}$ is derivable $\left(\mathrm{C}_{\mathrm{t}}\right.$ is a smooth curve of unit $\mathrm{t}$ as in Figure 4 or 5 ), though it is discrete mathematically. According to Conclusion (1), the derivative is negative. $\left(C_{t}-C_{0}\right)$ is the amount of "learning" effect, which will be zero if "learning" is no longer necessary. According to Conclusion (2), the absolute value of reduction rate should be proportional to $C_{t}-C_{0}$. Thus, we have,

$$
\frac{d\left(C_{t}-C_{0}\right)}{d t}=-k\left(C_{t}-C_{0}\right)
$$

Where $\mathrm{k}$ is a constant that reflects the percentage improvement amount over the unit. Solving the differential equation (2), we can obtain:

$$
\mathrm{C}_{\mathrm{t}}=\mathrm{C}_{0}+\left(\mathrm{C}_{1}-\mathrm{C}_{0}\right) \mathrm{e}^{-\mathrm{k}(\mathrm{t}-1)}
$$

This model can be used to estimate the resources or cost needed for each unit of the spacecraft under the impact of the nonlinear learning curve. $C_{1}$ is the recurring cost for the first unit that can be obtained from the estimate of the ST-5 project. $\mathrm{C}_{t}$ is the recurring cost for unit $t$ and $\mathrm{C}_{0}$ is the minimal standard cost of materials and labors needed for manufacturing the hardware that may be quoted from the manufacturers, such as how much it costs to order a sufficiently large quantity of products or subsystems. $\mathrm{C}_{0}$ can also be estimated through the regression model discussed below.

After many units have been produced or $t$ is a large number, $\left(C_{1}-C_{0}\right) e^{-k(t-1)}$ will be very small compared with $\mathrm{C}_{0}$. Then "learning" is nearly unnecessary and the recurring cost will be a standard cost $\mathrm{C}_{0}$. After ST-5 mission is completed, a linear regression analysis can also be performed to estimate $\mathrm{k}$ and verify $\mathrm{C}_{0}$. Taking a logarithm on Equation (3), we have

$$
\operatorname{Ln}\left(\mathrm{C}_{\mathrm{t}}-\mathrm{C}_{0}\right)=\operatorname{Ln}\left(\mathrm{C}_{1}-\mathrm{C}_{0}\right)-\mathrm{kt} \quad \text { or } \quad \mathrm{y}=\mathrm{a}-\mathrm{kt}
$$

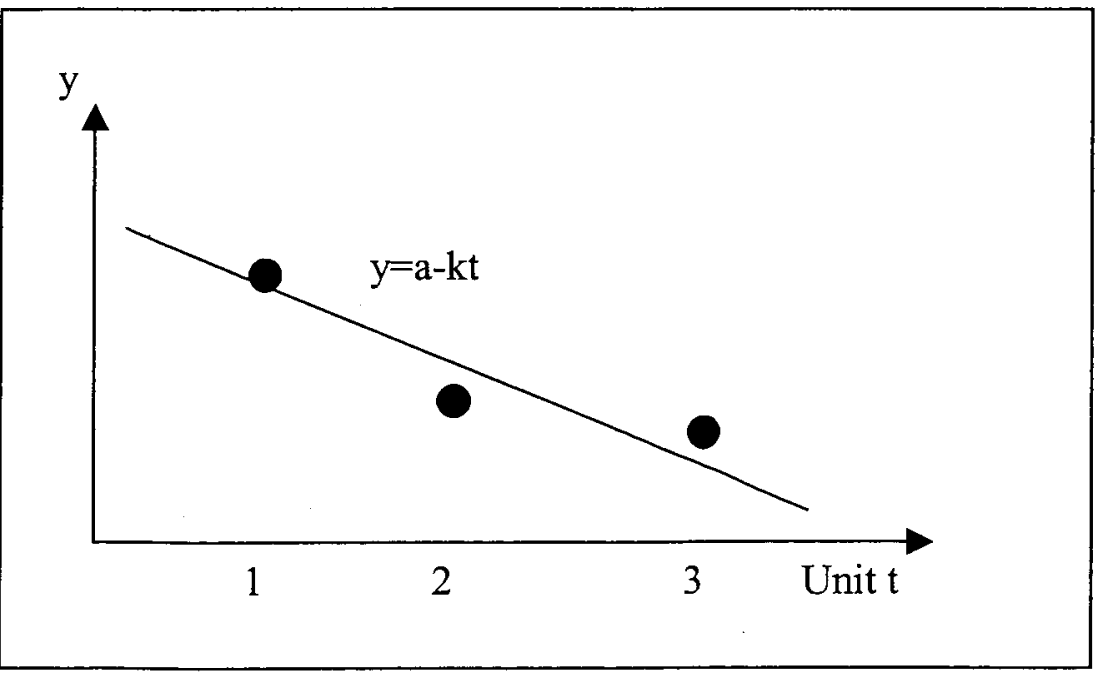

Figure 6. Regression analysis of the model. 
Where $\operatorname{Ln}\left(\mathrm{C}_{\mathrm{t}}-\mathrm{C}_{0}\right)$ is substituted by $\mathrm{y}$ and $\operatorname{Ln}\left(\mathrm{C}_{1}-\mathrm{C}_{0}\right)$ is substituted by a. The regression analysis can estimate the intercept and the slope of the linear model (4), and further to verify the estimated value of $\mathrm{C}_{0}$ (see also Figure 6). However, the cost data needs to be filtered to remove the cost impact of program delays (due to delays in acquisition of a launch vehicle). The impact should be categorized as nonrecurring cost.

According to Equation (1), in order to reduce the total cost, one of the most effective strategies is apparently, to reduce the recurring cost. This strategy pays off even at an expense of higher nonrecurring cost. For instance, to produce and fly 100 similar nano-satellites, a reduction in recurring cost can be reflected on the total cost by multiplying 100 times in saving. Examples include, standardizing the manufacturing of structures and common parts or components. To utilize the benefit of learning curve, we should build up the long-term reliable partnership with contract companies. We should try to reduce the recurring cost $b$ to a fixed necessary amount after several satellite production runs. For instance, ST- 5 project is a process of producing three pathfinder nano-satellites with a significant learning curve. The future production of 100 nanosatellites will occur at a very low recurring cost b. Thus, strategically, the cost of learning can be treated as a nonrecurring cost rather than as a recurring cost. That implies to use the same labor resources for manufacturing, integration as well as testing, and to build a long-term, dependable and trustful supplier or vendor-ship.

To reduce the total cost for multiple satellite development, apparently, reduction in recurring cost is much more effective than reduction in nonrecurring cost. Because standard production has lower cost, to reduce the recurring cost, we should standardize as many parts and components as possible. For instance, main structures can be produced by a standard mold or standard process. The power supplies and solar arrays can be produced as a standard module, etc. These considerations may require a higher nonrecurring cost because they include the cost for more molds, fixtures and modules. However, the return can be reflected on the reduction in recurring cost that can lower the total cost by $\mathrm{n}$ times, as specified in Equation (1). The trade between higher nonrecurring cost and lower recurring cost across all aspects of the project should be a key part of the conceptual planning phase of the project. It is clear that savings in recurring cost must be designed into the project and not forced in during development.

The duplicated and repetitive production will bring in the necessity of enhanced quality assurance to minimize the deviations from the desired performance. The concept of robustness in design and manufacturing may be useful. The performance of various spacecraft should be robust or insensitive to the undesirable manufacturing variations as well as predictable changes, such as a change in functional feature. Robust design is a systematic approach to quality [7]. Its ultimate goal is to build quality into products through good design. We should implement enhanced quality management according to aerospace standards in production. Of course, "do the job right at the first time" is very important. Wrong jobs will always bring waste in production, create loss and eventually add significantly to cost. While we consider cost reduction, quality, reliability and risk management must also be considered carefully in the multiple-spacecraft production mission. 


\section{CONCLUSIONS}

ST-5 project is a pathfinder for multiple-spacecraft constellation missions with validation of new ideas and new technologies in the space environment. We have discussed the impact of learning curve effect on the three ST-5 spacecraft, in terms of nonrecurring and recurring cost categorization. We have also proposed a model to predict the expenditures future multiple production runs of the identical spacecraft. A large part of ST-5 expenditures can be categorized as nonrecurring that includes concept development and design as well as design confirmation, plus a cost growth due to the launch vehicle slip because of the secondary payload dilemma. Since we may deal with multiple production runs (tens or hundreds) of satellites, optimal design and manufacturing techniques can lead to cost reductions while maintaining high-quality standards. Robustness can minimize the effects of variations in manufacturing, while having little impact on costs. Thus, we can achieve our goal for high quality and low production costs.

\section{Acknowledgement}

This research is supported in part by NASA Administrator's Fellowship Program and NASA Faculty Award for Research (FAR) grant NAG5-13365.

\section{References}

1) Evans, B. (editor): Satellite Communication Systems, 3rd edition, Institution of Electrical Engineers, London, UK, 1999

2) Bertheux, P., Douillet, F. and Chiarini, J. C.: A low-cost multi-mission bus for small satellite applications, Space Technology, Vol 18; pp. 171-174, 1998

3) NASA Goddard Space Flight Center, Concept Study Report for the New Millennium Space Technology 5 Small Satellite Constellation Theme, Goddard Space Flight Center, July 15, 1999.

4) Wertz J. and Larson, W., Space Mission Analysis and Design, Chapter $1,3^{\text {rd }}$ edn, Microcosm Press and Kluwer Academic Publishers, 1999.

5) Evans, J. R.: Applied Production and Operations Management, $4^{\text {th }}$ edition, West Publishing Company, 1993

6) Modern Technologies Corporation: Defense Manufacturing Management - Guide for Program Managers, Defense Systems Management College, Fort Belvoir, VA, April 1989.

7) Chen, G. and Oberright, J., "Integrating Robustness into ST-5 Nano-Satellite Development for Inexpensive Space Missions", an article in Smaller Satellites: Bigger Business? Space Studies Vol. 6 (edited by M. Rycroft and N. Crosby), Kluwer Academic Publishers, 2002. 